\title{
Resistance to immune checkpoint inhibitors. Next steps and combinational approaches
}

\author{
Thorsten Fuereder (D)
}

Received: 10 February 2019 / Accepted: 23 April 2019 / Published online: 17 May 2019

(C) The Author(s) 2019

\begin{abstract}
Summary Immuno-oncology and in particular checkpoint inhibitor (CPI) treatment has become a novel promising cancer therapy strategy in recent years. However, still a minority of patients respond to checkpoint blockade. Primary and secondary resistance to $\mathrm{CPI}$ is a challenge in the daily clinical routine. Combination strategies have been tested in various clinical trials in order to address this issue. Data available from these trials indicate improved activity depending on the tumor type. This review article focuses on the molecular background for resistance to CPI, gives an overview of current clinical data of CPI combination studies and points out potential strategies to overcome CPI resistance depending on the immune phenotype.
\end{abstract}

Keywords Immunotherapy · Checkpoint inhibitor · Primary resistance $\cdot$ Secondary resistance $\cdot$ Immune phenotype

\section{Introduction}

Immune checkpoint inhibitors (CPI) have entered the clinical routine during the last couple of years and CPI therapy is considered as the standard of care for a wide range of malignancies. A recent analysis of 262 patients treated with CPI and suffering from 19 different malignancies demonstrated an objective response rate (ORR) of $29 \%$ across all tumor types and a long-term survivor rate (i.e. longer than 2 years) of $11.8 \%$ [1]. Despite these advances, response to single agent CPI varies markedly between highly sensitive

\footnotetext{
T. Fuereder, MD ( $ه)$

Department of Internal Medicine I, Comprehensive Cancer Center, Division of Clinical Oncology, Medical University of Vienna, Währinger Gürtel 18-20, 1090 Vienna, Austria thorsten.fuereder@meduniwien.ac.at
}

tumors such as Hodgkin's lymphoma (ORR >65\%) and resistant ones such as microsatellite stable colorectal cancer (ORR $<10 \%$ ) [2, 3]. Apart from that, it has been observed that - while the majority of responses to CPI are durable-disease recurrence during immunotherapy or after (early) discontinuation occurs as reported in head and neck squamous cell carcinoma or nonsmall cell lung cancer patients $[4,5]$. Therefore, it is obvious that understanding the mechanisms of resistance to CPI therapy is crucial in order to turn nonresponsive tumors into responsive ones and prevent relapse during or after treatment with CPI. Overcoming resistance and rational combinatorial approaches have to be based on the molecular understanding of the underlying resistance mechanisms:

Resistance to CPI therapy can be divided into primary resistance (i.e. no upfront response to immunotherapy) and secondary (or acquired) resistance (i.e. after initial response to immunotherapy tumor recurrence/progression is observed) [6]. Both tumorintrinsic and -extrinsic (or host) factors can contribute to either primary or secondary resistance mechanisms [6].

\section{Primary resistance}

Primary resistance to CPI is partially based on adaptive mechanisms. Either the tumor is not recognized by the immune system at all or adaptive mechanisms facilitate immune escape [6]. As mentioned above various host and/or tumor intrinsic factors contribute to primary resistance. Host factors comprise the tumor microenvironment (TME), endocrine and metabolic factors, environmental factors such as dysbiosis or antibiotic or steroid use and other nonmodifiable factors such as age, chronic disease or unfavorable host genetics [7]. 


\section{Immune phenotype}

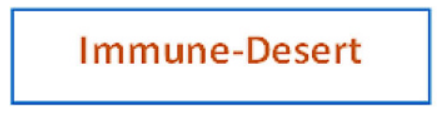

\section{Immune-Excluded}

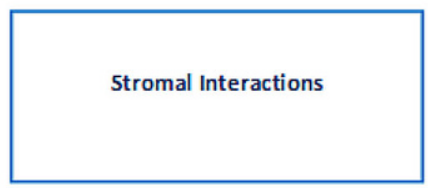

chemotherapy, radiation,

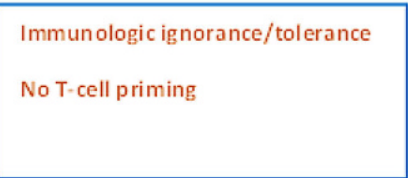

Potential combination strategies targeted therapy, other CPI

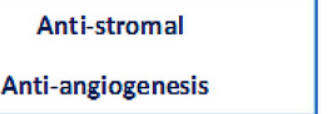

\section{Immune-Inflamed}

Exhausted T cells, suppressive cells

(Tregs, MDSC,..), cytokines

Alternative checkpoints

other CPI or immunomodulators

Fig. 1 Potential combination strategies of an immune checkpoint inhibitors (CPI) in order to overcome resistance based on the immune phenotype. MDSC myeloid-derived suppressor cells (Modified and adapted, [14, 25])

The complex interplay between the TME and the tumor itself determines if an effective antitumor immune response can take place. FoxP3+ T cells (Tregs) can dampen the immune response by direct cell-cell contact or the secretion of inhibitory cytokines such as IL-10, IL-35 or TGF-beta [6, 8]. Apart from that T-cell exhaustion, which is a state where $\mathrm{T}$ cells are exposed to continuous antigen signals and lose their effector function, contributes to primary resistance $[8,9]$. It was demonstrated that PDL-1 high exhausted CD8+ T cells respond poorly to PDL-1 blockade [10]. Besides T cells, myeloid-derived suppressor cells (MDSC) and tumor-associated M2 macrophages (TAMs) play a role in negative immune regulation [6, 8, 11]. Finally, adaptive upregulation of alternative immune checkpoints such as TIM-3 or Lag-3 was shown to be responsible for primary CPI resistance $[6,12]$.

Intrinsically, tumors can modulate their gene expression and pathways ultimately resulting in a lack of immune cell infiltration or suppression of an adequate antitumor immune response. Epigenetic modifications of immune-related genes or mutations in the interferon gamma pathway, the JAK pathway or constitutive PDL-1 expression also lead to a negative immune-response [8]. Additionally, low antigenicity of the tumor cell, which is mediated by a low tumor mutational burden or alterations in the antigen-presenting machinery, results in a lack of immune cell recognition [11].

\section{Secondary resistance}

Contrary to primary resistance, secondary resistance develops over time subsequent to constant pressure of the immune system on the tumor cells. Likewise, the immune-related gene expression profile of the tumor can be altered. Interferon gamma pathway mutations and loss of antigen presentation proteins leading to a lack of $\mathrm{T}$ cell recognition have been described [11]. In melanoma patients, who initially responded to CPI, but relapsed, a truncating beta 2 microglobulin mutation was detected by whole exome sequencing. This mutation resulted in the loss of surface expression of major histocompatibility complex class I [13].

\section{Immune phenotype}

Based on these considerations antitumor immunity can be classified into three main phenotypes as suggested by Chen and Mellman [14]: The inflamed tumor, the immune excluded tumor and the immune desert tumor. Each of this phenotype is associated with multiple mechanisms for primary/ secondary resistance to avoid response antitumor immune response [14]. While immune-desert tumors exhibit immunological ignorance, tolerance or lack of T-cell priming, stromal factors such as mechanical barriers, vascular factors or an immune-suppressive chemokine state are the predominate cause for immune evasion in immune-excluded tumors [14]. Finally, all types of mechanisms for resistance to immunotherapy described above can be detected in inflamed tumors. Translating these immune phenotypical findings into molecular signatures seems to be crucial for the elucidation of resistance. Although the pan cancer initiative, which analyzed more than 10,000 tumors in 33 cancer types employing comprehensive sequencing techniques, reported six types of immune signatures, which correlated with OS, the significance for predicting resistance to CPI or immunotherapy in general is still unclear [15]. 
Table 1 Selected combination trials in order to overcome resistance to CPI

\begin{tabular}{|c|c|c|c|c|c|c|}
\hline Author & Indication & Regimen & $\begin{array}{l}\text { Number of } \\
\text { Patients }\end{array}$ & HR for death & HR for progression/death & ORR \% \\
\hline $\begin{array}{l}\text { Wolchock } \\
\text { et al. [17] }\end{array}$ & $\begin{array}{l}\text { Untreated } \\
\text { advanced } \\
\text { melanoma }\end{array}$ & $\begin{array}{l}\text { Nivo and Nivo/lpi vs. Ipi } \\
\text { alone }\end{array}$ & 945 & $\begin{array}{l}\text { HR } 0.55,95 \% \text { Cl } 0.45-0.69 \\
\text { for Nivo/lpi vs. Ipi }\end{array}$ & $\begin{array}{l}\text { HR } 0.43,95 \% \mathrm{Cl} \\
0.35-0.52 \text { for Nivo/lpi } \\
\text { vs. Ipi }\end{array}$ & 57.6 for Ipi/Nivo \\
\hline $\begin{array}{l}\text { Motzer et al. } \\
\text { [18] }\end{array}$ & $\begin{array}{l}\text { Untreated ad- } \\
\text { vanced renal cell } \\
\text { cancer }\end{array}$ & Nivo/lpi vs. Sunitinib & 1096 & $\begin{array}{l}\text { HR } 0.63,99.8 \% \mathrm{Cl} \\
0.44-0.82\end{array}$ & $\begin{array}{l}\text { HR } 0.82,99.1 \% \text { Cl } \\
0.64-1.05 ; \text { not signifi- } \\
\text { cant }\end{array}$ & 42 for Ipi/Nivo \\
\hline $\begin{array}{l}\text { Motzer et al. } \\
\text { [19] }\end{array}$ & $\begin{array}{l}\text { Untreated ad- } \\
\text { vanced renal cell } \\
\text { cancer }\end{array}$ & Axitinib/Ave vs. Sunitinib & 886 & Final data not available yet & $\begin{array}{l}\mathrm{HR} 0.61,95 \% \mathrm{Cl} \\
0.475-0.790\end{array}$ & $\begin{array}{l}62 \text { for Axi- } \\
\text { tinib/Ave }\end{array}$ \\
\hline $\begin{array}{l}\text { Antonia et al. } \\
\text { [22] }\end{array}$ & Stage III NSCLC & $\begin{array}{l}\text { Chemorad/Durva vs. } \\
\text { Chemorad }\end{array}$ & 709 & HR $0.86,95 \%$ Cl $0.54-0.86$ & $\begin{array}{l}\text { HR } 0.52,95 \% \mathrm{Cl} \\
0.42-0.65\end{array}$ & $\begin{array}{l}28.4 \text { for } \\
\text { Chemorad/ } \\
\text { Durva }\end{array}$ \\
\hline $\begin{array}{l}\text { Socinski et al. } \\
\text { [24] }\end{array}$ & $\begin{array}{l}\text { Stage metastatic } \\
\text { non-squamous } \\
\text { NSCLC }\end{array}$ & $\begin{array}{l}\text { Chemo/Atezo/Bev } \\
\text { (and Chemo/Atezo) } \\
\text { vs. Chemo alone }\end{array}$ & 1202 & $\begin{array}{l}\text { HR 0.78, } 95 \% \text { Cl 0.64-0.96 } \\
\text { For EGFR/ALK: HR 0.54, } \\
95 \% \text { CI 0.29-1.03 (not } \\
\text { significant) }\end{array}$ & $\begin{array}{l}\text { HR } 0.62,95 \% \mathrm{Cl} \\
0.52-0.74\end{array}$ & $\begin{array}{l}63.5 \text { for Chemo/ } \\
\text { Atezo/Bev }\end{array}$ \\
\hline
\end{tabular}

\section{Next steps: combinatorial approaches to over- come resistance}

In order to overcome resistance to single agent CPI, combination strategies have been suggested and multiple trials testing CPI combinations are currently being conducted. Unfortunately, the landscape of clinical immunotherapy combination trials is quite often driven by the specific pipeline of the industry and does not always follow rational combination strategies based on the molecular and immune phenotype considerations outlined above [16]. Obviously, the vast majority of combination trials is conducted with the five approved CPI [16]. In general, several combination strategies of CPI with other compounds are tested in clinical trials. The most promising ones comprise the combination of a CPI with (a) another immunotherapeutic approach (vaccines; dual checkpoint blockade and removal of coinhibitory signals; activation of co-stimulatory signals; adoptive T-cell transfer; allogenic stem cell transplantation, ...), (b) DNA damaging agents such as cytotoxic chemotherapy or radiation or (c) targeted therapies (monoclonal antibodies or a tyrosine kinase inhibitors). Ideally, the distinct combination strategy employed should be tailored to the tumor microenvironment as summarized in Fig. 1.

\section{Clinical data}

Multiple clinical immunotherapy trials evaluating CPI combinations have been conducted so far (Table 1). Dual checkpoint blockade with a programmed cell death protein 1 (PD-1) antibody plus a cytotoxic T-lymphocyte-associated protein 4 (CTLA-4) targeting agent proved to be a successful strategy in melanoma, renal cell carcinoma and non-small cell lung cancer (NSCLC). A positive nivolumab plus ipilimumab trial has led to the approval of this com- bination in metastatic melanoma patients, since the risk for death was decreased with nivolumab plus ipilimumab compared to ipilimumab alone (HR 0.55, 95\% CI 0.45-0.69) [17]. Likewise it was demonstrated that in intermediate- or poor-risk advanced renal cell carcinoma patients, there was a significant improvement in OS with nivolumab plus ipilimumab compared to sunitinib (median not reached versus 26 months; HR $0.63,99.8 \%$ CI $0.44-0.82$ ) as demonstrated in the CheckMate 214 study, which resulted in FDA approval in this setting in 2018 [18]. Of note, dual checkpoint blockade significantly increases the rate of treatment-related adverse events and toxicity compared to single agent CPI therapy.

Overcoming primary resistance to CPI therapy can be achieved by the combination with targeted therapy. Axitinib plus the $\mathrm{PD}(\mathrm{L})-1$ inhibitor avelumab (or pembrolizumab) is superior to sunitinib monotherapy in advanced renal cell carcinoma (PFS 13.8 vs 8.4 months; HR 0.69) [19]. Although the tolerability of this combination was similar between the groups, it has to be noted that the combination of a CPI with targeted agent can lead to major toxicity as well. Doselimiting hepatotoxicity was observed for ipilimumab plus vemurafenib or nivolumab plus crizotinib for instance $[20,21]$.

A very interesting concept is the combination of radiotherapy and CPI. Radiotherapy triggers antigen release and might potentiate the efficacy of CPI therapy and turn an immune desert tumor into an inflamed one. Although the optimal radiation dose and fractionation is still under investigation, the PACIFIC study in stage III unresectable NSCLC patients, which evaluated platinum-based chemoradiation followed by checkpoint blockade via durvalumab vs. placebo, yielded positive results (HR for death $0.68,95 \%$ CI 0.54-0.86) [22].

Finally, cytotoxic chemotherapy plus CPI is an effective strategy in order to overcome immunother- 
apy resistance. Initial concerns that leukocyte depletion by chemotherapy might dampen the effect of CPI therapy turned out to be unjustified. On the contrary, numerous clinical trials such as the KEYNOTE189 study in NSCLC stage IV patients showed that chemotherapy plus CPI is superior to chemotherapy alone and not associated with a higher number of immune-related adverse events [23].

Even the combination of chemotherapy, antiangiogenic (targeted) therapy with bevacizumab plus CPI seems to be more effective that this combination without a CPI. The IMpower150 trial demonstrated a trend towards survival advantage for the addition of a CPI in EGFR-mutated or ALK-positive NSCLC stage IV patients, which used to be resistant to single agent checkpoint blockade (OS not reached compared to 17.5 months; HR $0.54 ; 95 \% \mathrm{Cl} 0.29-1.03$ ) [24]. However, the toxicity of this regimen is substantial and despite the OS benefit in this setting, the approach of a chemo/CPI/bevacizumab combination in the absence of an adequate biomarker seems questionable.

\section{Conclusion}

Primary and secondary resistance to single agent checkpoint blockade is an emerging problem in daily clinical routine given the increasing numbers of patients treated with immunotherapy. Knowledge about the reasons for resistance to immunotherapy is constantly expanding. Although results from immunotherapy combination trials are promising and resulted in approvals, future clinical immunotherapy combination trials should take the circumstances for resistance into account and select patients based on their specific host immune microenvironment.

Funding Open access funding provided by Medical University of Vienna.

Conflict of interest T. Fuereder is member of advisory boards and/or received honoraria from MSD, MERCK, Roche, Novartis, Astra Zeneca, BMS and Boehringer Ingelheim. Research funding by MSD and MERCK.

Open Access This article is distributed under the terms of the Creative Commons Attribution 4.0 International License (http://creativecommons.org/licenses/by/4.0/), which permits unrestricted use, distribution, and reproduction in any medium, provided you give appropriate credit to the original author(s) and the source, provide a link to the Creative Commons license, and indicate if changes were made.

\section{References}

1. Gauci ML, Lanoy E, Champiat S, et al. Long-term survival in patients responding to anti-PD-1/PD-L1 therapy and disease outcome upon treatment discontinuation. Clin Cancer Res. 2019;25:946-56.

2. Maly J, Alinari L. Pembrolizumab in classical Hodgkin's lymphoma. EurJ Haematol. 2016;97:219-27.
3. Le DT, Durham JN, Smith KN, et al. Mismatch repair deficiency predicts response of solid tumors to PD-1 blockade. Science. 2017;357:409-13.

4. Ferris RL, Blumenschein G Jr., Fayette J, et al. Nivolumab vs investigator's choice in recurrent or metastatic squamous cell carcinoma of the head and neck: 2-year long-term survival update of CheckMate 141 with analyses by tumor PD-L1 expression. Oral Oncol. 2018;81:45-51.

5. Salati M, Baldessari C, Cerbelli B, Botticelli A. Nivolumab in pretreated non-small cell lung cancer: continuing the immunolution. Transl Lung Cancer Res. 2018;7:91-4.

6. Sharma P, Hu-Lieskovan S, Wargo JA, Ribas A. Primary, adaptive, and acquired resistance to cancer immunotherapy. Cell. 2017;168:707-23.

7. Pitt JM, Vetizou M, Daillere R, et al. Resistance mechanisms to immune-checkpoint blockade in cancer: tumor-intrinsic and -extrinsic factors. Immunity. 2016;44:1255-69.

8. Bai J, Gao Z, Li X, Dong L, Han W, Nie J. Regulation of PD1/PD-L1 pathway and resistance to PD-1/PD-L1 blockade. Oncotarget. 2017;8:110693-707.

9. Wherry EJ, Kurachi M. Molecular and cellular insights into T cell exhaustion. Nat RevImmunol. 2015;15:486-99.

10. Blackburn SD, Shin H, Freeman GJ, Wherry EJ. Selective expansion of a subset of exhausted CD8 T cells by alphaPDL1 blockade. Proc Natl Acad Sci USA. 2008;105:15016-21.

11. Seto T, Sam D, Pan M. Mechanisms of primary and secondary resistance to immune checkpoint inhibitors in. Cancer Med Sci. 2019;7. https://doi.org/10.3390/ medsci7020014.

12. Oweida A, Hararah MK, Phan A, et al. Resistance to radiotherapy and $\mathrm{PD}-\mathrm{L} 1$ blockade is mediated by TIM-3 Upregulation and regulatory T-cell infiltration. Clin Cancer Res. 2018;24:5368-80.

13. Zaretsky JM, Garcia-Diaz A, Shin DS, et al. Mutations associated with acquired resistance to PD-1 blockade in melanoma. NEngl J Med. 2016;375:819-29.

14. Chen DS, Mellman I. Elements of cancer immunity and the cancer-immune set point. Nature. 2017;541:321-30.

15. Thorsson V, Gibbs DL, Brown SD, et al. The immune landscape of cancer. Immunity. 2018;48:812.

16. Tang J, Shalabi A, Hubbard-Lucey VM. Comprehensive analysis of the clinical immuno-oncology landscape. Ann Oncol. 2018;29:84-91

17. Wolchok JD, Chiarion-Sileni V, Gonzalez R, et al. Overall survival with combined Nivolumab and Ipilimumab in advanced melanoma. NEngl J Med. 2017;377:1345-56.

18. Motzer RJ, Tannir NM, McDermott DF, et al. Nivolumab plus Ipilimumab versus Sunitinib in advanced renal-cell carcinoma. NEngl J Med. 2018;378:1277-90.

19. Motzer. JAVELIN Renal 101: a randomized, phase 3 study of avelumab + axitinib vs sunitinib as first-line treatment of advanced renal cell carcinoma (aRCC). ESMO; 2018; Munich. 2018.

20. Ribas A, Hodi FS, Callahan M, Konto C, Wolchok J. Hepatotoxicity with combination of vemurafenib and ipilimumab. NEngl J Med. 2013;368:1365-6.

21. Spigel DR, Reynolds C, Waterhouse D, et al. Phase $1 / 2$ study of the safety and Tolerability of Nivolumab plus Crizotinib for the first-line treatment of Anaplastic Lymphoma Kinase Translocation-positive advanced non-small cell lung cancer (checkmate 370). J Thorac Oncol. 2018;13:682-8.

22. Antonia SJ, Villegas A, Daniel D, et al. Overall survival with Durvalumab after Chemoradiotherapy in stage III NSCLC. NEngl J Med. 2018;379:2342-50.

23. Gandhi L, Rodriguez-Abreu D, Gadgeel S, et al. Pembrolizumab plus chemotherapy in metastatic non-smallcell lung cancer. NEnglJ Med. 2018;378:2078-92. 
24. Socinski MA, Jotte RM, Cappuzzo F, et al. Atezolizumab for first-line treatment of metastatic Nonsquamous NSCLC. NEngl J Med. 2018;378:2288-301.

25. Duan J, Wang Y, Jiao S. Checkpoint blockade-based immunotherapy in the context of tumor microenvironment: opportunities and challenges. Cancer Med. 2018;7:4517-29.

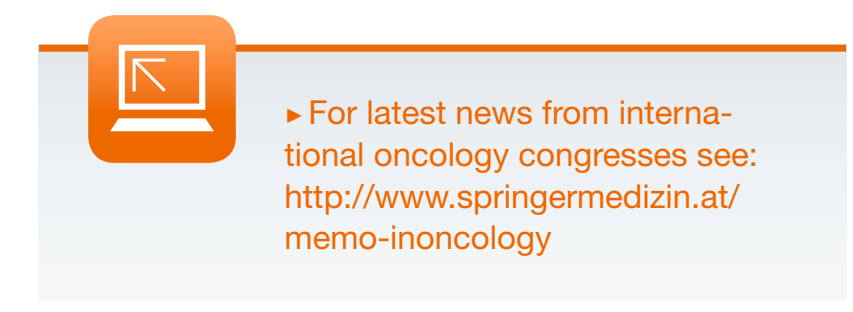

Publisher's Note Springer Nature remains neutral with regard to jurisdictional claims in published maps and institutional affiliations. 